\title{
A Brief Analysis on the Application of Minimalism in Book Cover Design —_A case study on Zhihong Wang the new-generation designer in Taiwan
}

\author{
Guohua Zuo \\ College of fine arts, Huanggang Normal University, Huanggang 438000, China
}

Keywords: Minimalism; Cover Design; Text; Graphics

\begin{abstract}
As with a unique style of design yielding the spirits of the times, minimalism has been highly advocated by many designers in the binding design of contemporary books, enjoying social penetration among the masses. Rather than making simple exclusion of the contents, minimalism requires the designers to take the essences and discard the dregs, that is, to refine and sublimate the contents for further creativity and presentation with a thorough understanding on the theme of design. Only in this way, can they be able to introduce a new visual feast to the audiences. Though there are certain discussions on the application of minimalism in the binding design of books at present, detailed and in-depth case studies are yet to fulfill. This paper will present a case study of the book cover design by Zhihong Wang, the famous new-generation designer in Taiwan, to elaborate the application of minimalism in the book cover design in the light of text, graphics and color design as an attempt to enhance deeper understanding of people on the elements of minimalism in book cover design.
\end{abstract}

\section{Introduction}

Minimalism, also known as simplicity, was first proposed by Richard Wollheim the aesthetic theorist in 1965, who advocated reducing the painting language down to colors and shapes by simplifying the picture by virtue of minimal use of colors and images and discarding any object that would distract the subject. [1] Evolved from modern thinking of design prevailing at the beginning of the twentieth century, minimalism directs artistic creation in the principle of reducing to the minimal. The concept of less is more, put forward by Ludwig Mies van der Rohe the famous architect in 1930s, advocates simple design against excessive decoration, is thereby respected as the core idea of minimalism. Featured by maximum simplicity on the elements, lighting and raw materials, nevertheless, minimalism is demanding for colors and materials. Therefore, it can always achieve a great effect with less and simple use of objects. Although originated in the West, it has been long shining its spirits in the ancient Chinese culture. According to Tao Te Ching by Lao Zi, the words “万物之始, 大道至简, 衍化至繁” reveal the fact that all creatures at the beginning are born simple before growing into a mess of complexity, while the words “少则得, 多则惑” emphasize simplicity and the importance of a macro picture. According to Confucius, the line “大 乐必易、大礼必简” tell us that the best music and etiquette always favor simplicity, while in Legend of Ma Yuan in The History of Late Han Dynasty, the word 简约 (simplicity)taken from the line that describes how the Queen Empress was leading a plain life with everything simplified does stresses being simple and thrifty on self-cultivation and in life, thereby embodying the ideas of returning to nature and tracing back to origins. In this sense, it can be seen that both the idea of less is more in the West and the ideology of simplicity of ancient Chinese scholars have shared the same. That is, it is the design community in the West that strongly pushes the style of minimalism to the climax, while ancient Chinese scholars put stress on the idea from all aspects of human life. 


\section{The Application of Minimalism in Book Cover Design}

Zhihong Wang, a famous graphic designer in Taiwan, has created a series of excellent works on book cover since 2000. The author is to discuss the application of the minimalism in the design of book cover from the following three aspects.

(1) Text Design being Novel, Unique, Open and Ethereal

In the binding design of modern books, the design of the text, the core of reading, has played a pivotal role, and the design of its connotation counts for a great importance. As for book binding design, elements of text design, including font size and arrangement, not only determine on the external aesthetic feeling but also bridge up the readers, books and designers, while it can be said that the design of text connotation lies at the heart of it. The success of Zhihong Wang should be paid attribute to the novel and unique text design, which has made well combination of the connotation of the book and the simple design of the text title.

Take his work of book cover design for the book Shifangyinian for instance. The words Shi (ten) and Yi (one) are amplified to serve as the title, while the four words Shifangyinian are downsized and placed in the upper left corner and the name of the author Lin $\mathrm{Xi}$ in the upper right corner. Because of low color brightness, the words Shi(ten) and Yi(one) look like the signs of plus and minus from afar, arousing a sense of intricate fancy. On the whole, the text design is concise and simple, weaving a feeling related to vanity of Zen as open and ethereal to the edifying effect. Thanks to its simple and novel design, it further arouses people`s attention and reflection over the idea of simple design.

The design for The Sixth Ward is clever. Incomplete and seemingly morbid glyphs of 5 and 7 are presented on the left and right sides of the cover respectively to indicate a 6 in the middle that represents the ward, which is intriguingly shown on the back invisibly. In the upper right corner and the lower right corner of the figure 5, there are the Chinese and English name of the title and author of the book respectively, enabling the integration of the idea of simplicity, clever conception and careful reservation of vacant space. This indeed can refer to the delicate arrangement of layout and structure.

Based on the two works of book cover design for Shifangyinian and The Sixth Ward, it can be seen that the text design of Zhihong Wang has been kept keen and novel. He believes that a strong appeal to text design has been accumulated from consciousness to actual graphic design. Referring to his years of exploration, he also has found that text can bring in a greater shock for a specific design. Given the fact that image publishing design has been prevailing for more than ten years in the mainstream while the approach of pure text design is physically scarce, he personally feels that the two have been in a state of imbalance and more emphasize should be paid to the latter.

Zhihong Wang has ever pointed out in an interview that it is necessary to understand the shapes of text at hand and the proper method to handle with if to achieve a good text design. Herein we shall take the text title for the cover of Empire of Signs for instance to see his processing techniques. The Chinese characters 符号 (sign) are placed as two parallel rows, which are separated into parts and further made into black embossing. As the embossing looks like slight depression, one can only see the separated parts from afar. Such creativity quite resembles the yin and yang fish in an eight-diagram, being both novel and simple. Though there is two circles in red and gray color overlapped in the middle of the cover with some text explanation below, it does not reduces the effect of simplicity on the whole.

(2) Simple and Elegant Graphic Design with Deep Connotation

In the book cover design, graphics play a significant role in conveying the connotation of the work. To select graphics, it should be customized according to the contents, market background, and the style of the author and requirements of the customer without any blind patchwork. Featured as simple and symbolic, graphics are able to directly reflect the main content of a book and convey the information in a fast and intuitive manner. A simple layout design eloquent to express the theme should be the most shocking and persuasive in book layout design. Standard varies from time to 
time. As minimalism is the product of the times, it shall be marked with the stamp of the age. [4] As far as his works are concerned, he can always extract one or more highlights from the contents of a book and further generalize into graphics, thereby reflecting the connotation of the book and sticking closely to the subject of the times. In an interview with him regarding the book cover design of the book A Chinese Font Walk, he has mentioned that though graphics are more easy to digest, it will be overwhelmingly direct and prevent the readers from thinking, and that's why he less emphasizes on the use of photos. That's why he adopts the symbolic graphics other than traditional images in his book cover design without hesitation.

For You are the April of this World: the Complete Works of Lin Huiyin, he goes the other way of traditional tedious design, highlighting a sense of youth, modernity and design while maintaining the feminine elegance and spirituality. Six symbols including the star, rain, cloud, water, snow and flower are generalized with curves and polylines to set against the feminine elegance and temperament, while graphic symbols are designed in white to elegantly dot around. The cover adopts a simple beige color, in the company of concise graphics and texts which are designed based on the grid line system of architecture, serving as a foil to the grace charm of the book.

In the cover design of Journal de Deuil, he only uses the graphics of two drops of tears, with one in pale gray under the Chinese words 哀悼 (mourning) as hints of sadness and the other in dark gray under 日记 (diary) as grief countless to exhaust a pen. Though with a few text and graphics of two drops of tears in gray, it is exactly concise and ethereal and makes a reading process interesting. In the contemporary book cover design, the style of minimalism has been gaining more and more attention from designers and consumers, and will become a trend of the times beyond doubts.

(3) Color Design being Pure, Bright, Young and Lively

Eton, the Swiss painter who has taught at the Bauhaus Academy in Germany, ever says that color is power [5]. Indeed, color is of special importance in visual arts. In particular, in book cover design, unique color application turns out to be more appealing and stimulate the desire to make a purchase. To a great extent, the formation of the style of book cover design depends on the color individuality. It is difficult to imagine how a cover design caters for the aesthetic pursuit and artistic taste without any color individuality in modern times. A book cover design of minimalism generally bears on the features of primitive simplicity and elegance. Minimalism pursues for color simplicity and brightness and advocates color individuality, therefore in the process of color application, simple color combination is always favored by designers and audiences.

Simplicity has always been with the good works by excellent designers. Only by those book designers equipped with profound artistic skills and visual communication skills can we see those works in simple forms, elegant colors and deep connotation. In addition to be in simple and concise form, minimalism also emphasizes the use of pure colors, that is, to adopt similar color map in the light of hue, brightness and purity so as to make the works more delicate and superimposed. For the cover design of Yayoi Kusama, he uses the contrast colors of red and blue with a red dot matrix arranged along the oblique line in the background, creating a sense of order and high-tech. The English title is presented in blue oversized letters, giving a cool and pure sense, while the body in black and white and Japanese words on the below are particularly elegant, reflecting a kind of graceful and simple beauty. On the whole the color tone of the cover is young, playful and vibrant. With respect to color, the style of minimalism also advocates being refined, simple and streamlined and returning to the origin of design, so as to explore the essences of design in the most popular and simple manner to achieve the most primitive artistic effect. In this way, it demonstrates the philosophical speculation of designers and their understanding on the meaning of life, and wakes up the primitive and chaotic memories in people.

\section{Conclusion}

As a green and humanized design, the style of minimalism has been in the mainstream of modern book cover design, both catering for the contemporary fast-food style of life and greatly stimulating the desire to purchase and read books. With his cover design following the trend, Zhihong Wang 
has been ranked among the top Chinese in cover design. What matters is that he elaborates in the processing of text, graphics and color, showing a strong capability in the use of minimalism. It is the very fact of that he has developed the design style of minimalism that sticks closely to the times and the aesthetic feeling of modern people that enables his works turn out to be great success and enjoy extensive popularity.

\section{References}

[1] Fangfang Han, Study on the Visual Language of Modern Books under the Concept of Minimalism [D] Zhejiang Agriculture and Forestry University .2013: 6-7

[2] Xiangna Lee,Weiqiang Wu. The Design Concept and Aesthetic Value of Apple Product from the Perspective of Marxist Huumanism [J] Journal of Wuhan University of Technology (Section of Social Science) 2015 (4): 613-614

[3] Litao Sun. On the Analysis of the Occasions and Methods for the Use of Ballad and Proverb of the Han Dynasty [J] Chinese Culture Forum .2014 (5): 69-70

[4]Ying Gao. On the Idea of Minimalism in Packaging Design [J] Decoration .2007 (9): 116-117

[5] Jinlin Zou, Yiqing Peng. On the Impact of Color Individuality on the Design Style of Book Cover[J] Art Panorama. 2011 (8): 106-107

[6] Hong Xu. Minimalism in Graphic Design [J] Grand Stage .2011 (10): 114-115 